\title{
An Economic and Environmental Assessment Model for Microchannel Device Manufacturing: Part 1 - Methodology
}

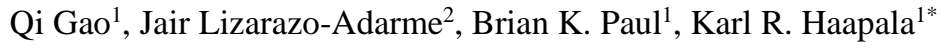 \\ ${ }^{1}$ School of Mechanical, Industrial, and Manufacturing Engineering, 204 Rogers Hall, Oregon State University, \\ Corvallis, OR, USA 97331 \\ ${ }^{2}$ Battelle/Pacific Northwest National Laboratory, Microproducts Breakthrough Institute, 1000 NE Circle Boulevard, \\ Suite 11101, Corvallis, OR, USA 97330
}

\begin{abstract}
Wordcount: 7,266

To manage the effects of manufacturing industries on the economy and environment, design tools are needed that will aid engineers to assess manufacturing costs and environmental impacts early in design. The development of cost analyses and environmental assessments can be burdensome, but much of the data used in these analyses can be shared. Little work has been done to integrate environmental assessments with cost analyses for the purposes of evaluating manufacturing plans for both environmental impact and cost. A spreadsheet-based cost and environmental impact assessment model is developed for simultaneously analyzing the effects of manufacturing process plans on both product cost and the environment. A bottom-up process-based cost calculation method is used to estimate microchannel device manufacturing costs over a range of production volumes, while process-based cradle-to-gate life cycle assessment is utilized to evaluate environmental impacts. Both models draw data from a common database for describing the device geometry and the production environment. The method is used to evaluate the manufacturing plan for a microchannel heat exchanger, providing insight into the likely drivers of cost and environmental impact. For this case study, the analysis shows that both cost and environmental impact are dominated by the same process step - diffusion bonding.
\end{abstract}

Keywords: Bottom-up cost modeling, Environmental impact assessment, Decision making, Microchannel process technology, Graphical user interface

\section{List of Abbreviations}

$\begin{array}{ll}\text { EDM } & \text { Electrical Discharging Machining } \\ \text { EI } & \text { Environmental Impact } \\ \text { GUI } & \text { Graphical User Interface } \\ \text { HRU } & \text { Heat Recovery Unit } \\ \text { LCA } & \text { Life Cycle Assessment } \\ \text { LCI } & \text { Life Cycle Inventory } \\ \text { LCI } & \text { Life Cycle Impact Assessment } \\ \text { PCM } & \text { Photochemical Machining } \\ \text { VBA } & \text { Visual Basic for Applications }\end{array}$

\section{Introduction}

Low manufacturing cost is considered one of the most essential elements in product design and process planning (Allen, 2004; Roy et al., 2004; Weinert et al., 2004). Economical manufacturing development, however, often has resulted in environment problems and concerns (Gutowski et al., 2005). In recent years, environmental considerations have gained public interest and are entering into manufacturing decisions. To reduce the environmental impacts of manufacturing, researchers have investigated the environmental aspects of various processes. Two decades ago, Munoz and Sheng (1995) and Choi et al. (1997) investigated approaches to improve the environmental performance of conventional manufacturing processes. More recently, Anastaselos et al. (2011) and Bradley et al. (2006) considered environmental impact as a factor in decision support modeling. Research has analyzed process environmental impacts from macro (Fratila, 2010) and micro levels. Several studies have investigated micromanufacturing processes. For example, Liow (2009) studied the energy consumption of micromilling, Modica et al. (2011) researched micro 
electrical discharging machining (micro EDM), and Brown et al. (2011) and Haapala et al. (2009) studied the environmental impacts of microprocessing technologies for the production of a microchannel device. Several researchers have demonstrated the potential to achieve both cost-efficient and environmentally-friendly manufacturing through the choice of cutting fluid (Tan et al., 2002), the use of optimized process parameters (Winter et al., 2014), and the development of manufacturing techniques (Allen and Jefferies, 2006), among other approaches. Design tools have not been demonstrated, however, to enable the comparison of different manufacturing alternatives for both cost and environmental impact. To improve the sustainability of manufacturing operations, these types of design tools are needed (Haapala et al., 2013).

Zhao et al. (2012) proposed a method to develop process plans where manufacturing cost and environmental impact are considered simultaneously for process candidates. This method did not consider the effect of production volume on cost and environmental impact, however; thus the results cannot accurately represent the impacts at different scales of production. Eastlick and Haapala (2012) developed a sustainability assessment method for product design where economic performance, environmental impacts, and social effects were considered for different metrics. This work also focused on the product design process, and did not consider the effects of production volume. Avram et al. (2010) and Zhang and Haapala (2014) developed methods to incorporate sustainability assessment into manufacturing decision making where economic, environment, and social aspects are addressed. The approaches involved multicriteria decision making for a single set of processes for machining metal products.

To address the limitations of prior approaches, a combined economic and environmental assessment method for modeling production facilities is introduced herein. Cost assessment is based on bottom-up, process-based cost modeling (Lajevardi et al., 2011; Leith et al., 2010). Environmental impact assessment differs from conventional, product-focused methods, and uses a manufacturing-oriented process-based life cycle assessment (LCA) method to evaluate impacts based on cost model categories of capital tooling, capital facilities, utilities, consumables, and raw materials. Thus, the method simultaneously estimates unit manufacturing costs and environmental impacts over a range of production volumes. A graphical user interface (GUI) is used to simplify the collection of data used to support cost and environmental impact analyses. The GUI enables rapid evaluation of process alternatives through sensitivity analysis. Detailed calculations for estimating costs and environmental impact are described in the next section, along with a description of the data requirements. An application of the model is demonstrated by analyzing the process plan for a microchannel heat exchanger.

\section{Assessment Modeling Methodology}

The economic and environmental impact assessment model developed in this research is intended to estimate the overall manufacturing costs and environmental impacts of alternative process flows at a range of production volumes. Production and manufacturing process parameters, tool information, and product geometry are required for the model calculations. The algorithms for calculating the costs and environmental impacts are presented below.

\subsection{Cost model}

A process-based cost calculation method is utilized to build the cost model. Process-based cost modeling is a bottomup cost calculation method, which has been applied in prior work (Lajevardi et al., 2011; Leith et al., 2010). Total cost is broken down into different cost categories, and the cost associated with each category is calculated based on the associated process steps. As illustrated in Figure 1, categories include tool, facility, labor, maintenance, raw materials, consumables, and utilities costs, which are discussed in greater detail below. 


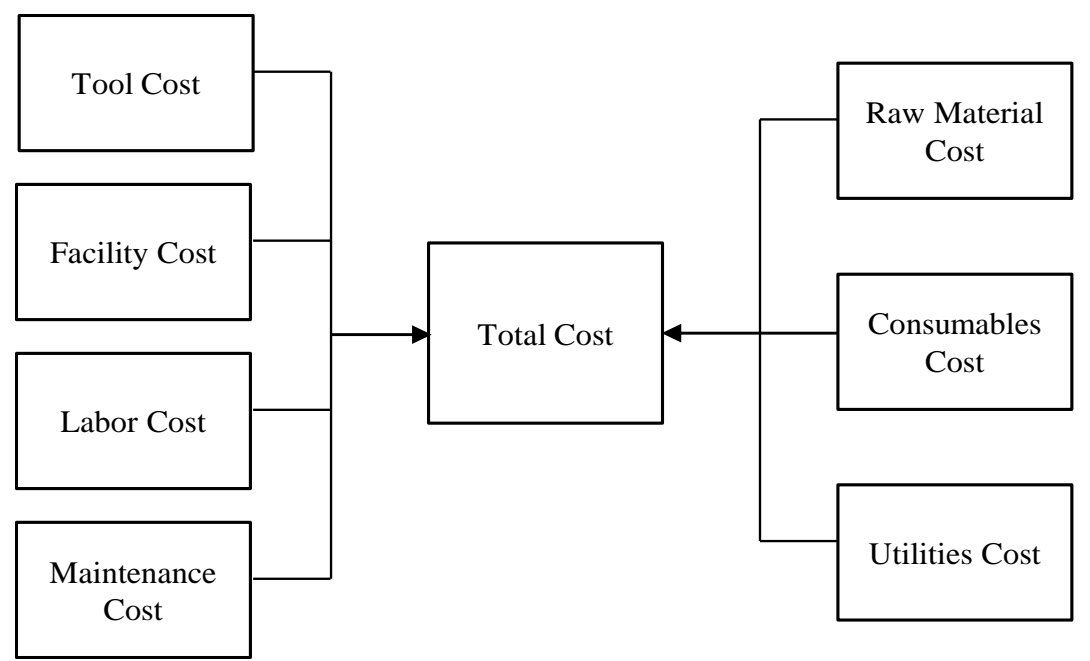

Figure 1. Contributors to product manufacturing costs

\subsubsection{Tool cost}

Tool (equipment) cost includes the capital costs and installation costs of the equipment required to perform the manufacturing operations. Since the capital cost is depreciated over the lifetime of the tool, the cost of one tool (one unit of equipment) is allocated to each of the parts processed by that tool during its operational lifetime. Thus, to calculate cost, assume an annual production volume, $P$, requires $n$ tools of type $j$ working simultaneously. Let $t_{j}$ represent the lifetime of tool $j$ in years. Then $n$ times the sum of unit tool capital cost, $C_{\text {tool }}$, and unit tool installation cost, $C_{\text {installation, }}$ gives the total tool cost. To allocate the cost to each part processed by the tools, the total cost is divided by the total number of parts processed over $t_{j}$ years. This calculation is illustrated in Eq. 1, where $C_{T}$ refers to the tool cost per part.

$$
C_{T}=\frac{\left(C_{\text {tool }}+C_{\text {installation }}\right) \times n}{t_{j}{ }^{* P}}
$$

To determine the number of tools, $n$, required for a given production volume, information about the production capacity and tool capacity is needed. To meet the annual production volume, $P$, with every tool working $k$ hours per year, hourly production capacity of the system is $P / k$. Tool capacity, the number of parts processed per tool per hour, can then be determined by knowing the cycle time, $t_{c}$ (number of hours for one part to be processed), step yield, $y$ (fraction of acceptable parts produced), and the tool utilization, $u$ (Eq. 2). Since the number of tools is an integer value, the result of the calculation is rounded up to the nearest integer.

$$
n=\left\lceil\frac{P / k}{y \times\left(1 / t_{c}\right) \times u}\right\rceil
$$

If a process has several steps and each step requires multiple tools, the cost equation can be changed to (Eq. 3):

$$
C_{T}=\sum_{i=1}^{a} \sum_{j=1}^{b_{i}} \frac{\left(C_{\text {toolij }}+C_{\text {installation }}\right) \times n_{i j}}{t_{i j} \times P}
$$

where $i$ is the number of steps in the process $(i=1,2,3, \ldots, a)$ and $j$ is the number of tools used in each step $(j=1,2$, $3, \ldots, b_{i}$, where the value of $b_{i}$ depends on the nature of each step). Note that $n_{i j}$ is calculated based on cumulative yield, $Y_{i j}$, and the probability of success for a series of steps is based on the success of each step and the success of each tool within each step. Thus, let $y_{i j}$ be the yield of each successive step and $Y_{i j}$ be the cumulative yield as (Eq. 4): 


$$
Y_{i j}=\prod_{i=1}^{a} \prod_{j=1}^{b_{i}} y_{i j}
$$

\subsubsection{Facility cost}

Facility cost refers to facility construction cost and is allocated to the parts processed over the lifetime of the facility $\left(\mathrm{t}_{\text {fac }}\right)$. The cost of manufacturing space is $\mathrm{C}_{\mathrm{mfg}}$ per unit area $\left(\$ / \mathrm{m}^{2}\right)$ and the space required for each tool is $S_{\text {tool }}\left(\mathrm{m}^{2}\right)$. To allocate the facility cost to each part processed over the facility lifetime, the total facility cost is divided by the number of parts processed over the life of the facility (Eq. 5).

$$
C F=\frac{C_{m f g} \times S_{\text {tool }} \times n}{t f a c \times P}
$$

The floor space required includes the actual footprint of each tool ( $\mathrm{S}_{\text {tool_act }}$ ), as well as space needed for workers to operate around it ( $\mathrm{S}_{\text {tool_work }}$ ). Therefore, $S_{\text {tool }}$ can be calculated as Eq. 6.

$$
\text { Stool }=\text { Stool_ }_{-} \text {act }+ \text { Stool }_{-} \text {work }
$$

If a process has several steps and each step requires multiple tools, Eq. 7 can be used,

$$
C_{F}=\sum_{i=i}^{a} \sum_{j=1}^{b_{i}} \frac{C_{m f g} \times S_{\text {tooli }} \times n_{i j}}{t_{f a c} \times P}
$$

where $i$ is the number of steps of a process and $j$ is the number of tools used in step $i$.

\subsubsection{Labor cost}

Labor cost $\left(\mathrm{C}_{\mathrm{L}}\right)$ is calculated for each process based on the annual salary of manufacturing personnel working on tool, $C_{S}$, the loaded labor cost rate, $R$, the number of personnel needed to staff the tool, $n_{s}$, the annual production volume, $P$, and the number of tools needed to meet the production volume, $n$. Loaded labor rate accounts for employee hourly rate plus employee fringe benefits. Total labor cost is allocated to each part processed, as shown in Eq. 8.

$$
C_{L}=\frac{C_{s} \times R \times n_{s} \times n}{P}
$$

If the process has $i$ steps and each step requires $j$ tools, this equation can be modified as follows (Eq. 9):

$$
C_{L}=\sum_{i=1}^{a} \sum_{j=1}^{b i} \frac{C s_{i j} \times R_{i j} \times n_{s i j} \times n_{i j}}{P}
$$

\subsubsection{Tool maintenance cost}

Periodic service is needed to maintain the tools (equipment) in operating condition, including inspection, problem detection, and failure correction. In this model, the maintenance cost is assumed to be a fraction, $r_{m}$, of tool capital cost. Thus, per part tool maintenance cost can be calculated as (Eq. 10):

$$
C M=\frac{C \text { tool } \times n \times r m}{P}
$$

If the process has $i$ steps and each step requires $j$ tools, this equation can be modified as follows (Eq. 11): 


$$
C_{M}=\sum_{i=1}^{a} \sum_{j=1}^{b_{i}} \frac{C_{t o o l i j} \times n_{i j} \times r m_{i j}}{P}
$$

\subsubsection{Utilities cost}

Utilities cost, $C_{U}$, includes the cost of water, electricity, and wastewater (Eq. 12). Water use cost is determined from the hourly water use per tool, $n_{W}$, water unit cost, $C_{W}$, and cycle time, $t_{c}$ (hours). Similarly, electricity use cost is determined from the hourly electricity use per tool, $n_{E}$, electricity unit cost, $C_{E}$, and cycle time. Wastewater charges are determined from the amount of wastewater generated, $n_{w w}$, wastewater charges per unit, $C_{W W}$, and cycle time. If other utilities are used in the process, e.g., natural gas, they can be added to the model accordingly.

$$
C U=(C W \times n w+C E \times n E+C W W \times n w w) \times t_{c}
$$

If the process has $i$ steps and each step requires $j$ tools, this equation can be modified as follows (Eq. 13):

$$
C U=\sum_{i=1}^{a} \sum_{j=1}^{b_{i}}\left(C W \times n W_{i j}+C E \times n E_{i j}+C W W_{i j} \times n W W_{i j}\right) \times t_{C i j}
$$

\subsubsection{Consumables cost}

Consumables are materials used in production that facilitate production and may become a part of the final product. The cost of a consumable $c, C_{c}$, is calculated according to its unit price, $C_{c}$, and the amount used, $n_{c}$. This cost can vary among the different processes and even among steps in one process. The amount required can be calculated either based on the size of the device or, for consumables such as cutting gas or metalworking fluid, the amount used is related to the process cycle time, $t_{c}$, and the consumable consumption rate, $r$. In this case, $n_{c}$ can be calculated as $r \times t_{c}$. The cost of a consumable can be expressed by Eq. 14 .

$$
C_{C}=C_{c} \times n_{c}
$$

If a process has $i$ steps and each step requires $j$ tools with $c$ consumables applied $\left(c=1,2,3, \ldots, d_{i j}\right)$ in each step, the equation can be modified as follows (Eq. 15):

$$
C_{C}=\sum_{i=1}^{a} \sum_{j=1}^{b_{i}} \sum_{c=1}^{d_{i j}} C_{c i j} \times n_{c i j}
$$

\subsubsection{Raw material cost}

Raw materials are materials used in production that form all or a portion of the final product. In this model, raw material cost, $C_{R}$, is calculated based on unit price, $P_{R}$, and amount used $n_{R}$. It is assumed that the unit price is not affected by the amount of raw material use. If $i$ types of raw materials are used $(i=1,2,3, \ldots, a)$, total raw material cost can be expressed by Eq. 16 .

$$
C R=\sum_{i=1}^{a} P_{R i} \times n R i
$$

\subsection{Environmental impact model}

LCA is a method to quantitatively analyze the environmental impacts of products and systems (Goedkoop et al., 2009). Generally, there are four steps in LCA, which include defining the study goal and scope, conducting an inventory analysis, conducting an environmental impact assessment, and interpreting the results (ISO, 2006, p. 200). The purpose of the environmental impact model is to assist users in estimating and analyzing cradle-to-gate (material acquisition and production) environmental impacts of products. In this work, the functional unit for cost and 
environmental impact comparison is one microchannel device. From the process-based manufacturing information used in the cost analysis (e.g., user-defined device geometry and production rate), a list of mass and energy inputs is obtained, forming the life cycle inventory (LCI) for the LCA.

To generate environmental impact assessment values from the LCI data, a commercially available software tool (SimaPro 7) is used. The ReCiPe 2008 method is used to characterize and normalize environmental impacts. ReCiPe 2008 is a recently developed life cycle impact assessment (LCIA) method reported by Goedkoop et al. (2009). The method quantifies environmental impacts and reports the results in points, where one point represents the impact equivalent to one thousandth of the annual load of a person living in Europe. As addressed by Goedkoop et al. (2009), there are three perspectives available when choosing to weight impact assessment results: the Hierarchist, Egalitarian, and Individualist perspectives. These perspectives reflect the differences in value systems, and place varying importance on different damage types (i.e., human health, ecosystem quality, and resource depletion). In this work, the environmental impact model calculates the overall scores using the manufacturing-related categories considered in cost analysis: tool, facility, utilities, consumables, and raw material, so that environmental impact assessment and cost analysis can be conducted and interpreted simultaneously. The algorithms used are described below.

\subsubsection{Tool environmental impacts}

The environmental impacts of one tool (one unit of equipment) are allocated to each of the parts processed by that tool during its operational lifetime. As stated above, an annual production volume, $P$, requires $n$ tools of type $j$, where $n$ can be calculated using Eq. 2. Assume tool $j$ is made of $h$ types of material $\left(h=1,2,3 \ldots, e_{j}\right)$ with a mass of $m_{j h}$ and a unit impact score of $I_{j h}(\mathrm{Pts} / \mathrm{kg})$, and let $t_{j}$ represent the lifetime of the tool $j$ in years. The environmental impact score of tool $j$ allocated to one device at a given production volume can be calculated as shown in Eq. 17.

$$
I T_{j}=\frac{\sum_{h=1}^{e_{j}} m_{j h} \times I_{j h} \times n}{t_{j} \times P}
$$

If a process has $i$ steps and each step requires $j$ tools, the equation can be modified as (Eq. 18):

$$
I T=\sum_{i=1}^{a} \sum_{j=1}^{b_{i}} \frac{\sum_{h=1}^{e_{i j}} m_{i j h} \times I_{i j h} \times n_{i j}}{t_{i j} \times P}
$$

\subsubsection{Facility environmental impacts}

Facility environmental impacts are the environmental impacts resulting from facility construction and are allocated to the parts processed during the lifetime of the facility ( $\left.\mathrm{t}_{\mathrm{fac}}\right)$. The environmental impact score of manufacturing space is $I_{m f g}$ per unit area $\left(\mathrm{Pts} / \mathrm{m}^{2}\right)$ and the space required for each tool is $S_{\text {tool }}$ (Eq. 6). To allocate the facility environmental impacts to each part processed over the facility lifetime, the total impact score is divided by the number of parts processed over the facility lifetime (Eq. 19).

$$
I F=\frac{I m f g \times S t o o l \times n}{t f a c \times P}
$$

If a process has $i$ steps and each step requires $j$ tools, the equation can be modified as (Eq. 20):

$$
I_{F}=\sum_{i=i}^{a} \sum_{j=1}^{b_{j}} \frac{I_{m f g} \times S_{\text {toolij }} \times n_{i j}}{t_{f a c} \times P}
$$




\subsubsection{Utilities environmental impacts}

Utilities environmental impacts, $I_{U}$, include the impacts of water, wastewater, and electricity (Eq. 21). The water and wastewater impact score is determined by the hourly water use per tool, $n_{W}$, wastewater per tool, $n_{W W}$, water and wastewater unit impact scores, $I_{W}$ and $I_{W W}$, and cycle time, $t_{c}$. Similarly, electricity environmental impacts are determined from the hourly electricity use per tool, $n_{E}$, electricity unit impact score, $I_{E}$, and cycle time. If there are other utilities used in the process, e.g., natural gas, they can be added to the model accordingly.

$$
I U=(I W \times n W+I E \times n E+I W W \times n W W) \times t_{c}
$$

If a process has $i$ steps and each step requires $j$ tools, the equation can be modified as (Eq. 22):

$$
I U=\sum_{i=1}^{a} \sum_{j=1}^{b_{j}}\left(I W \times n W_{i j}+I E \times n E_{i j}+I W W \times n W W_{i j}\right) \times t_{c i j}
$$

\subsubsection{Consumables environmental impacts}

The environmental impacts of a consumable, $c$, are calculated according to its unit impact score, $I_{c}$, and the amount used, $n_{c}$. Impacts can vary among the different processes and even among steps in one process. The amount used can be calculated either based on the size of the device or, for consumables like cutting gas or metalworking fluid, the amount used is related to the process cycle time, $t_{c}$, and the consumable consumption rate, $r$. In this case, $n_{c}$ can be calculated as $r \times t_{c}$. The environmental impacts for a consumable can be expressed by Eq. 23 .

$$
I_{C}=I_{c} \times n_{c}
$$

If a process has $i$ steps and each step requires $j$ tools with $c$ consumables applied $\left(c=1,2,3, \ldots, d_{i j}\right)$, the equation can be modified as (Eq. 24):

$$
\text { IC }=\sum_{i=1}^{a} \sum_{j=1}^{b_{i}} \sum_{c=1}^{d_{i j}} I_{c i j} \times n_{c i j}
$$

\subsubsection{Raw material environmental impacts}

In this model, the raw material environmental impact score, $I_{R}$, is calculated based on unit impact score, $I_{r}$, and amount used $n_{R}$. If $i$ types of raw materials are used $(i=1,2,3, \ldots, a)$, total raw material environmental impacts can be expressed by Eq. 25.

$$
I_{R}=\sum_{i=1}^{a} I r_{i} \times n R_{i}
$$

\subsection{Modeling interface}

The above cost and environmental impact algorithms are realized in a MS Excel spreadsheet-based model, which contains three sets of worksheets: model inputs, calculations, and results. Input worksheets include a Production and Design Inputs sheet, where production parameters and product geometry are recorded; a Process Flow Inputs sheet, where manufacturing process parameters are recorded; and an Environmental Impact (EI) Inputs sheet, where environmental impact parameters are stored. Calculation worksheets include the Process Calculation sheet, where all the cost calculations are contained, and an EI Calculation sheet where all environmental impact calculations are performed. Results worksheets include the Process Results sheet and the EI Results sheet, where the cost and environmental impact results are reported, respectively, in tabular and graphical form. 


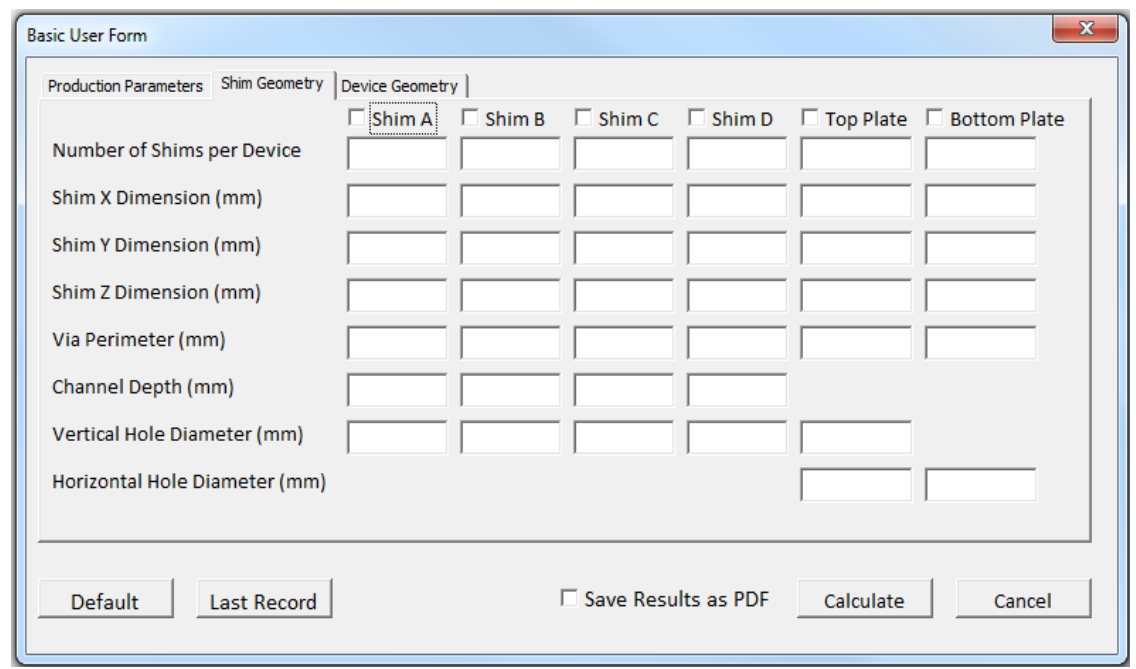

Figure 2. Graphical user interface for shim geometry data entry

A GUI was designed and implemented using MS Visual Basic for Applications (VBA) to facilitate data entry for modeling microchannel device manufacturing costs and environmental impacts. The GUI is composed of a user form with three tabs: a Production Parameters tab, a Shim Geometry tab, and a Device Geometry tab. In the Shim Geometry tab, shown in Figure 2, users are able to input information about the shim designs (a microlaminated device is created by layering up thin metal sheets, or shims, of various dimensions). In the Production Parameters tab, see Appendix A, Figure A.1, users are able to enter the expected minimum and maximum annual production volumes, while in the Device Geometry tab, see Appendix A, Figure A.2, users are able to input information about the interconnect surface. Using the GUI, the user can recall default settings by clicking the Default button, or recall the previous analysis conducted by clicking the Last Record button.

Cost and environmental impact results are calculated automatically when the Calculate button is clicked. A report is generated in .pdf format if the Save Results as PDF checkbox is checked when calculations are performed. Otherwise, the results are viewed in the model MS Excel file. By inputting the range of annual production volumes for evaluation, the model will calculate nine intermediate data points as potential annual production volumes, and generate the cost and environmental impact scores for each of these scenarios. Cost and environmental impact results for the nine production scenarios are reported for each of the cost categories. To assist a process analysis, cost and environmental impacts of each process are also shown for the seven categories.

\section{Application of the Modeling Methodology}

The manufacturing of a microchannel heat recovery unit (HRU) is chosen to illustrate the simultaneous assessment of cost and environmental impact using the above modeling methodology. Microchannel devices can be used as heat exchangers to capture the waste heat due to the small working distances within microscale channels, which facilitate rapid heat and mass transfer (Mehendale et al., 2000). Diesel engines generate a large amount of waste heat, resulting in a significant amount of energy loss. A cross-flow HRU can be used to move waste heat from hot exhaust gases into a secondary oil flow with low pressure drop. The heated oil can then be used to transport the energy for use in other applications. By using microchannels, the efficiency of heat transfer is increased leading to a compact HRU compared to conventional heat exchanger designs. In the HRU, stainless steel laminae, or shims, are machined with two different microchannel patterns, interleaved into a stack, and then bonded to form a monolithic device. This process architecture is termed microchannel lamination (Paul and Peterson, 1999).

The HRU consists of two shim configurations (Figure 3) - one guides the exhaust air flow (exhaust, or air, shim) and the other guides the oil flow (oil shim). The HRU is a composed of 46 air shims (210 $\mathrm{mm} \times 140 \mathrm{~mm} \times 0.9906 \mathrm{~mm}$ ) and 45 oil shims $(210 \mathrm{~mm} \times 140 \mathrm{~mm} \times 0.3048 \mathrm{~mm})$. The channel depths are $0.8 \mathrm{~mm}$ and $0.15 \mathrm{~mm}$ for the air shims and oil shims, respectively. Two holes are designed on each shim with a diameter of $11.68 \mathrm{~mm}$. After being bonded into a monolithic device, the holes form a channel to guide the oil flow. Additional microchannels are designed on oil 
shims so that rapid heat exchange can be realized. The HRU utilizes top and bottom plates $(210 \mathrm{~mm} \times 140 \mathrm{~mm} \times 12.7$ $\mathrm{mm}$ ), which each contain four $6.35 \mathrm{~mm}$ diameter holes for interconnecting the device with the vehicle exhaust system.
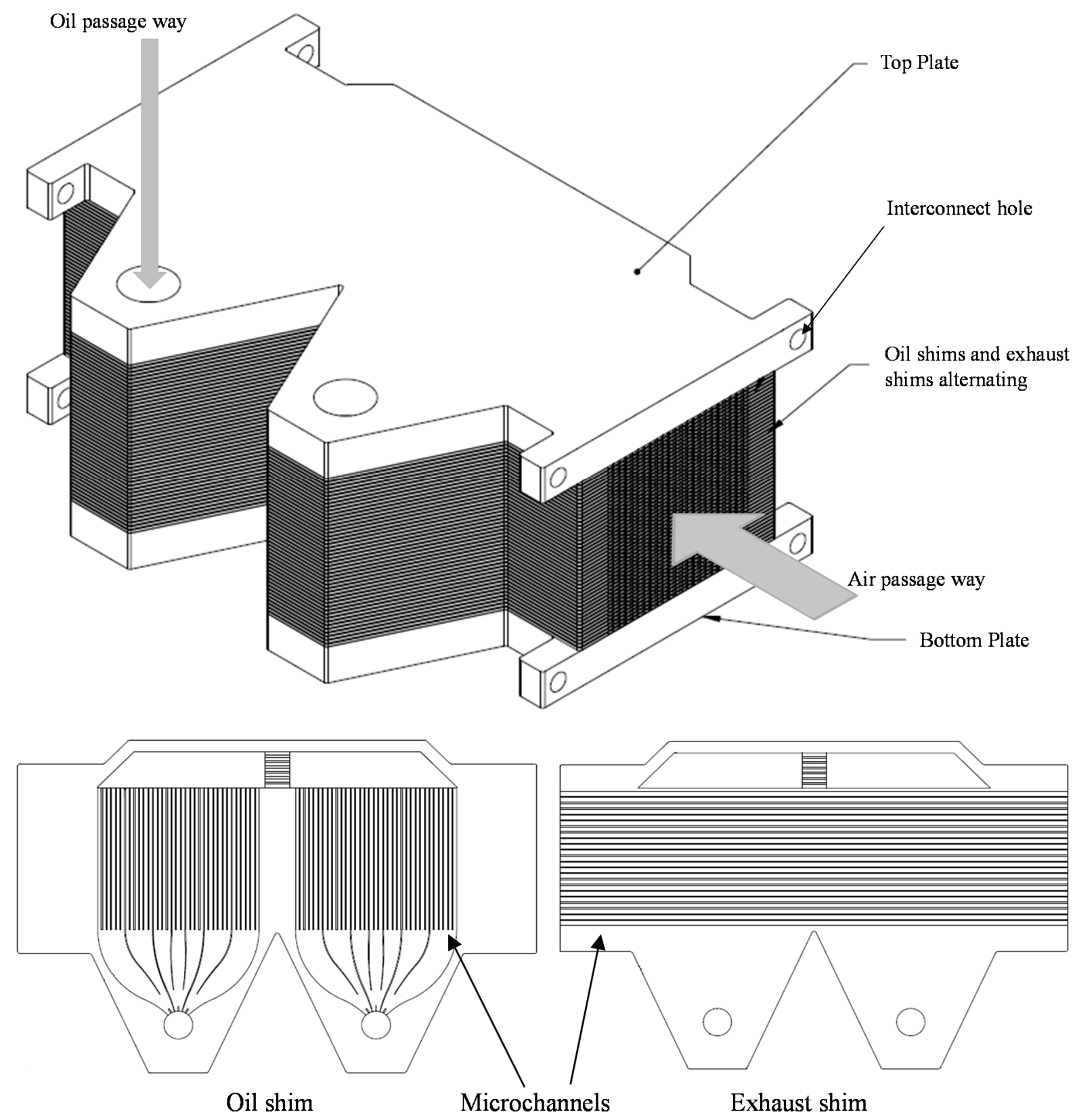

Figure 3. Heat recovery unit (HRU) design

To produce the device, photochemical machining (PCM) is used to etch the microchannels and holes into the shims. PCM starts with an alkaline and acid cleaning process in order to achieve a clean surface condition for etching. The model assumes that the whole surface of the stainless steel panel needs to be cleaned. Alkaline and acid chemical usage is assumed to be $0.11 \mathrm{~L} / \mathrm{m}^{2}$. The panels are then heated to lamination process temperature. Photoresist dry films and photomasks are laminated to the cleaned substrate and exposed to ultraviolet light to harden the selected area. Chemicals are sprayed to clean the unexposed area and develop the pattern. Then, a chemical solution is sprayed onto the sheet to etch the pattern in metal shim. The etch rate is assumed to be $0.1 \mathrm{~mm} / \mathrm{min}$. Next, the remaining photoresist 
is stripped, followed by a visual defect inspection to check the quality of the product. Finally, the metal etchant waste is reclaimed and treated as a part of the process flow.

Diffusion bonding is assumed to be used to join the shims and end plates together. Alkaline and acid cleanings are needed at the beginning of the process for degreasing and particulate removal. The cleaned panels are stacked and aligned in a fixture and bonded. Depending on the panel dimensions, the panels will form one or more devices. During the bonding process, panel stacks are heated to a bonding temperature of $980^{\circ} \mathrm{C}$ at a heating rate of $5{ }^{\circ} \mathrm{C} / \mathrm{min}$. The total bonding time is $42.7 \mathrm{hrs}$., with a cooling rate of $0.23^{\circ} \mathrm{C} / \mathrm{min}$., a holding time of $120 \mathrm{mins}$., and a furnace loading time of 30 mins. The bonding process yield is assumed to be $75 \%$ percent. Due to the difference between the stock panel size and device design dimensions, a singulation process is needed to cut the bonded panel stacks and endplates to the final dimensions of the HRU. Water jet cutting is assumed to be used to singulate (separate) the device to its final geometry. The linear cutting rate of water jet cutting is assumed to be $50 \mathrm{~cm} / \mathrm{min}$. per millimeter of laminae thickness. Computer numerical control (CNC) machining with a $6.35 \mathrm{~mm}$ diameter endmill is assumed to be completed at a feed rate of $500 \mathrm{~mm} / \mathrm{min}$. and a cutting depth of $3.175 \mathrm{~mm}$ to create the interconnect holes in the endplates. EDM is assumed to be used to finish the mating surfaces with an assumed linear cutting rate of $30 \mathrm{~cm} / \mathrm{min}$. per millimeter of laminae thickness. The general manufacturing process flow for the HRU is illustrated in Figure 4.

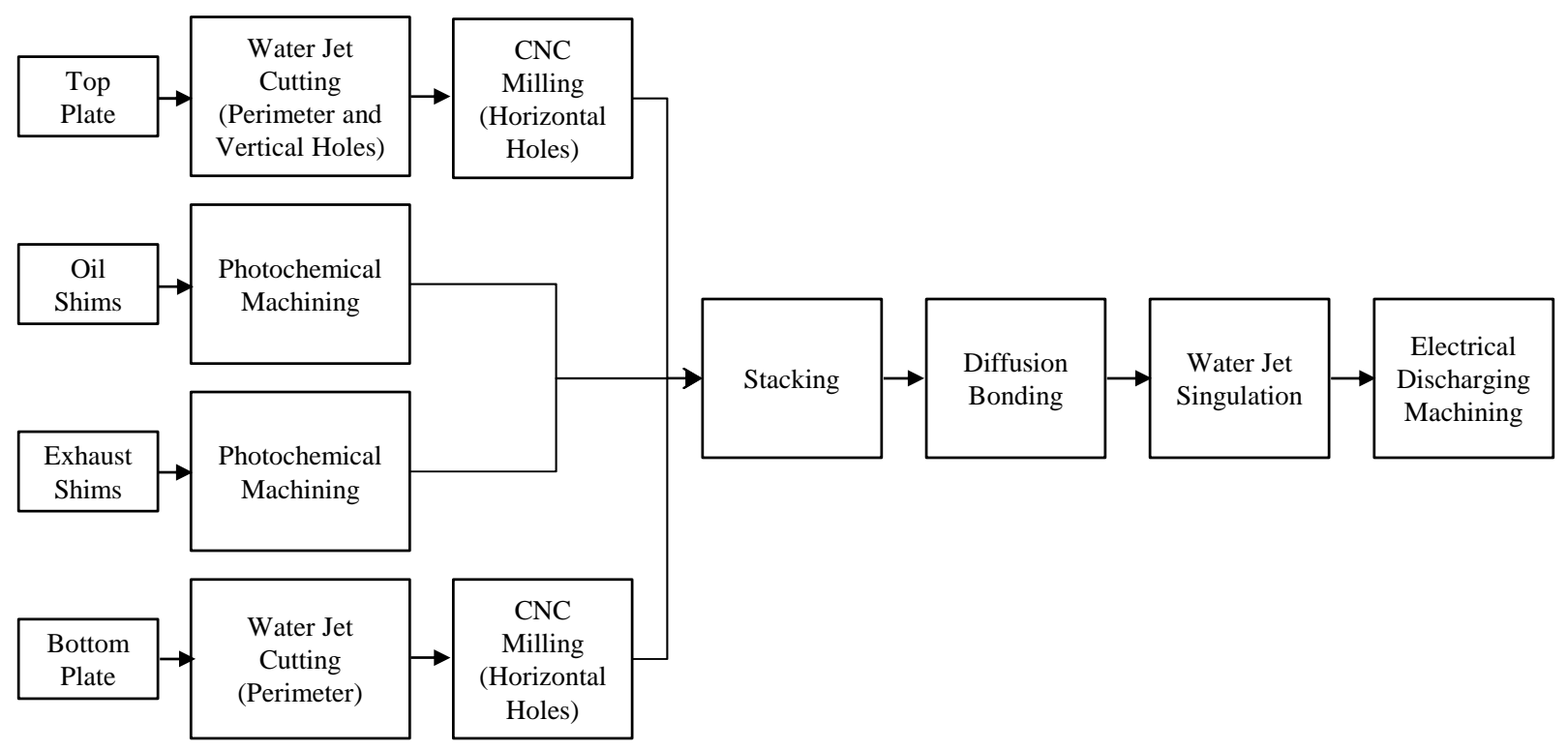

Figure 4. General manufacturing process flow for the heat recovery unit (HRU)

To perform modeling, a piece of equipment (tool) is selected for each process step. Tool installation cost is assumed as $4-5 \%$ of tool capital cost, yield for each process step is assumed to be $95-100 \%$, tool utilization is assumed to be $90-95 \%$, maintenance cost is assumed to be $3-5 \%$ of tool capital cost, and tools are assumed to be depreciated over seven years. For each tool, the facility space needed is assumed to be twice the actual footprint of the tool. Tools are assumed to operate 24 hours per day for 360 days per year. The facility depreciation period is assumed to be 25 years. Labor cost is assumed to be $\$ 40,000$ per year, based on the average salary rate of a Manufacturing Technician II in Portland, OR (Salary.com, 2013).

All process parameters, such as cycle time and tool capital costs, are determined based on information from equipment vendors. Due to the lack of information about tool material composition, for the life cycle inventory it is assumed that tools consist of iron and steel. For tools without available mass information, a material density of $150 \mathrm{~kg} / \mathrm{m}^{2}$ of footprint is assumed. Although uncertainties surround expendable tool components, such as the filter for the EDM tool and the nozzle and pump internals of the water jet cutter, these parts are neglected in environmental impact modeling. The inventory of material and energy inputs is given in Table 1 . Utility assumptions, including a water price of \$0.004/gal. (\$0.001/L) (Portland Water Bureau, 2013), wastewater and sewer charges of \$0.015/gal. (\$0.004/L) (Environmental Service, 2013), and electricity cost of $\$ 0.035 / \mathrm{kWh}$ (Portland General Electric, 2013) are obtained based on the average rates for industrial users in Portland, OR in 2013. 
Table 1. Inventory of material and energy inputs for each process

\begin{tabular}{|c|c|c|c|}
\hline Process Name & Category Name & Material/Energy Type & SimaPro Process Model \\
\hline- & Raw material & 316 Stainless steel & X5CrNiMo18 (316)I \\
\hline \multirow{9}{*}{ PCM } & Tool & Iron and steel & Iron and steel, production mix \\
\hline & Facility & Building & Building, Hall/CH/I \\
\hline & \multirow[b]{2}{*}{ Utilities } & Water & Water, deionized \\
\hline & & Electricity & $\begin{array}{l}\text { Electricity, medium voltage, } \\
\text { production }\end{array}$ \\
\hline & \multirow{5}{*}{ Consumables } & $\begin{array}{c}\text { Sodium hydroxide } \\
\text { (3\%, } 4 \%, 25 \% \text { solutions) }\end{array}$ & $\begin{array}{l}\text { Sodium hydroxide } \\
\text { (concentrated) E }\end{array}$ \\
\hline & & $\begin{array}{c}\text { Hydrochloric acid } \\
(10 \%, 15 \%, 30 \% \text { solutions })\end{array}$ & Hydrochloric acid, $30 \%$ in $\mathrm{H}_{2} \mathrm{O}$ \\
\hline & & $\begin{array}{c}\text { Polymethyl methacrylate } \\
\text { PET film }\end{array}$ & $\begin{array}{l}\text { Polymethyl methacrylate, sheet } \\
\text { PET film (production only) E }\end{array}$ \\
\hline & & Sodium carbonate & $\begin{array}{l}\text { Sodium carbonate from } \\
\text { ammonium chloride production }\end{array}$ \\
\hline & & $\begin{array}{l}\text { 40\% Ferric chloride } \\
45 \% \text { Sodium chlorate }\end{array}$ & $\begin{array}{l}\text { Iron (III) chloride, } 40 \% \text { in } \mathrm{H}_{2} \mathrm{O} \\
\text { Sodium chlorate, powder }\end{array}$ \\
\hline \multirow{6}{*}{$\begin{array}{l}\text { Diffusion } \\
\text { Bonding }\end{array}$} & Tool & Iron and steel & Iron and steel, production mix \\
\hline & Facility & Building & Building, Hall/CH/I \\
\hline & \multirow[b]{2}{*}{ Utilities } & Water & Water, deionized \\
\hline & & Electricity & $\begin{array}{l}\text { Electricity, medium voltage, } \\
\text { production }\end{array}$ \\
\hline & \multirow{2}{*}{ Consumables } & 25\% Sodium Hydroxide & $\begin{array}{l}\text { Sodium hydroxide } \\
\text { (concentrated) E }\end{array}$ \\
\hline & & 15\% Hydrochloric acid & Hydrochloric acid, $30 \%$ in $\mathrm{H}_{2} \mathrm{O}$ \\
\hline \multirow{7}{*}{$\begin{array}{l}\text { Water Jet } \\
\text { Cutting }\end{array}$} & Tool & Iron and steel & Iron and steel, production mix \\
\hline & Facility & Building & Building, Hall/CH/I \\
\hline & \multirow[b]{2}{*}{ Utilities } & Water & Water, deionized \\
\hline & & Electricity & $\begin{array}{l}\text { Electricity, medium voltage, } \\
\text { production }\end{array}$ \\
\hline & \multirow{3}{*}{ Consumables } & Garnet abrasive & Abrasive products \\
\hline & & Nozzle & N/A \\
\hline & & Pump internals & N/A \\
\hline \multirow{5}{*}{ EDM } & Tool & Iron and steel & Iron and steel, production mix \\
\hline & Facility & Building & Building, Hall/CH/I \\
\hline & \multirow[b]{2}{*}{ Utilities } & Water & Water, deionized \\
\hline & & Electricity & $\begin{array}{l}\text { Electricity, medium voltage, } \\
\text { production }\end{array}$ \\
\hline & Consumables & $\begin{array}{c}\text { Filter } \\
\text { Brass wire }\end{array}$ & $\begin{array}{c}\text { N/A } \\
\text { Brass }\end{array}$ \\
\hline \multirow{5}{*}{ CNC } & Tool & Iron and steel & Iron and steel, production mix \\
\hline & Facility & Building & Building, Hall/CH/I \\
\hline & \multirow[b]{2}{*}{ Utilities } & Water & Water, deionised \\
\hline & & Electricity & $\begin{array}{l}\text { Electricity, medium voltage, } \\
\text { production UCTE }\end{array}$ \\
\hline & Consumables & Carbide cutting tool & $\begin{array}{l}\text { Ferrochromium, high-carbon, } \\
68 \% \mathrm{Cr}\end{array}$ \\
\hline
\end{tabular}




\subsection{Cost modeling for the HRU}

For this illustrative example, the minimum and maximum annual production volumes are chosen to be 1,000 and 500,000 HRUs, respectively, giving the nine production scenarios of 1,000, 2,000, 5,000, 10,000, 20,000, 50,000, $100,000,200,000$, and 500,000 units. For an annual production of 1,000 HRUs, the cost of each device is estimated as $\$ 1,521.65$. As the production volume increases from 1,000 to 20,000 units, the cost of each device decreases tremendously (52.13\%), from $\$ 1,521.65$ to $\$ 728.42$ (Fig. 5a), as labor and equipment utilization increases. As the production volume increases from 20,000 to 500,000, manufacturing cost of each device continues to decrease, but is comparatively constant (\$728.42 to \$700.37) suggesting improved labor and equipment utilization. As the production volume increases, tool, facility, utilities, maintenance, and labor costs decrease significantly. The model assumes the raw material and consumable unit costs are not dependent upon production volume, which is reasonable since these are commodities. Figure 5b illustrates the cost breakdown by process type and raw materials. It can be seen that the cost for each process decreases with increasing production rate, while raw material costs are constant. The singulation and interconnect processes have the least significant impact on cost, compared to the bonding and patterning processes.

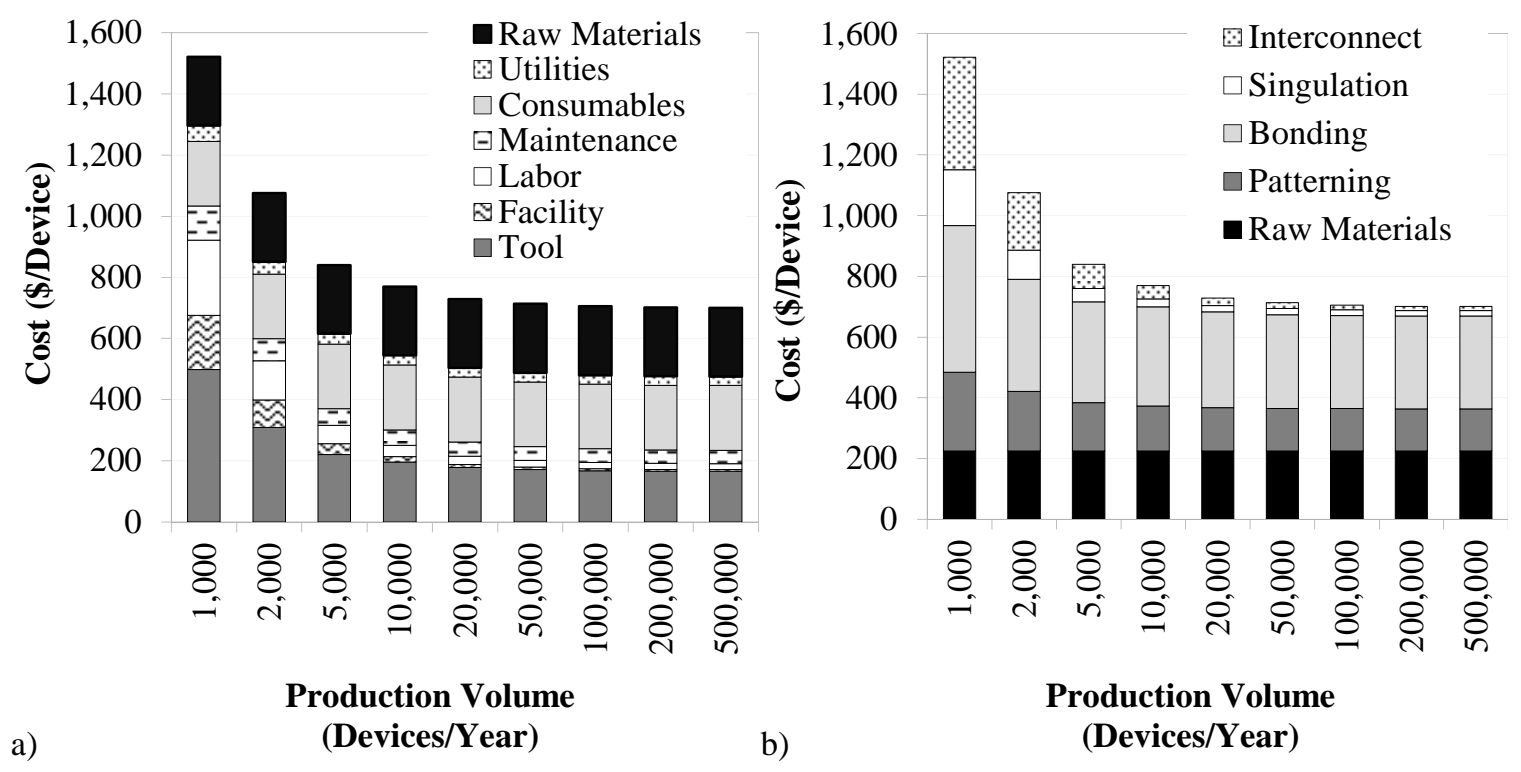

Figure 5. Cost breakdown by a) Cost category and b) Process type and raw material

Figure 6 illustrates this more clearly, where the cost for each process is broken down into the selected cost categories for a production volume of 20,000 HRUs per year, at which point costs stabilize. In the figure, cost categories with a fraction of total cost less than $5 \%$ are not numbered. The major contributors to patterning process costs are consumables, in particular photoresist and etchant reclaim, which are not affected by production volume. Because the cost of consumables per device is not dependent on production volume, changes in the total cost of the patterning process only reflect changes in other cost categories, which are comparatively minor, resulting in the relative insensitivity of the patterning cost. For the diffusion bonding process, the majority of the cost consists of tool capital costs. This is not only because of the high capital cost of diffusion bonding tool, but also because of the long bonding times leading to low tool capacities and the need for a large number of tools. For the singulation process, which is done using water jet cutting, the majority of cost is for consumables, while for the interconnect process, which uses EDM, the majority of the cost is related to the tool. As production volume increases, the costs associated with each tool are allocated across a larger number of devices, the cost of each device decreases.

\subsection{Environmental impact modeling for HRU}

Environmental impact assessment is performed for the nine production volume scenarios introduced above. Similar to cost, environmental impacts per unit show a decreasing trend from low production volume to high production volume, from 303.34 Pts at 1,000 HRUs per year to 295.16 Pts at 500,000 HRUs per year. This reduction (2.70\%) is considered relatively minor, however, due to the uncertainties in the analysis. Environmental impact results for a 
production rate of 500,000 HRUs per year are shown in Figure 7, which illustrates the environmental impact score breakdown by impact category of key importance; utilities contribute $87.8 \%$ of the impacts. Raw material and consumables, with impact scores of 19.20 Pts and 16.58 Pts, rank second and third, respectively. Cycle time is the variable that affects the environmental impacts of utilities, and is little sensitive to the change in production volume.

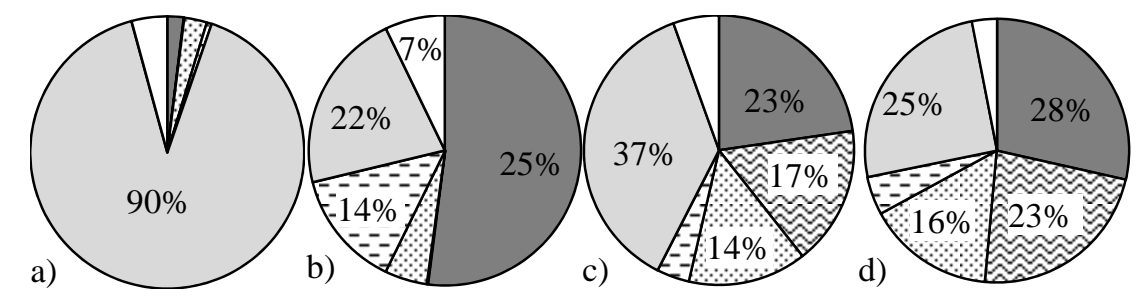

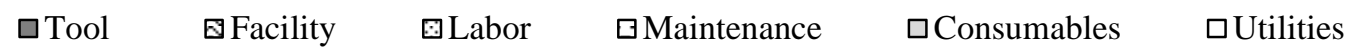

Figure 6. Key cost categories for a production volume of 20,000 devices/year: a) Patterning, b) Bonding, c) Singulation, and d) Interconnect processes

The model assumes the environmental impact scores of raw materials and consumables are not dependent upon production volume. Therefore, environmental impact scores of utilities, raw material, and consumables are constant at all production rates. Thus, changes in the total environmental impacts only reflects changes in other impact categories, namely tools and the facility. Since tools and the facility account for less than $1 \%$ of the total score, the environmental impacts per device are insensitive to changes in production volume. Figure $7 \mathrm{~b}$ exhibits the environmental impact score breakdown by process type. It can be seen that diffusion bonding accounts for $85.2 \%$ of the total process impacts. This is because of the nature of diffusion bonding, in which the laminated metal plates are heated to a high temperature for a long bonding time which requires a large amount of cooling water and electricity. The patterning process and raw materials, with impact scores of 19.58 Pts and 19.20 Pts, respectively, account for $6.63 \%$ and $6.51 \%$ of impact. Figure 8 illustrates this more clearly, where the environmental impact score for each process is broken down according to the different category types. Categories with a fraction of environmental impacts less than $5 \%$ are not numbered. As shown in the figure, the major contributor to patterning process environmental impacts is consumables, due to the large amount of consumables used in patterning, while the impact of utilities is also significant. Utilities account for $99.4 \%$ of total bonding impacts. For singulation and interconnect processes, utilities are again the major contributor, while consumables are a significant driver for both processes.
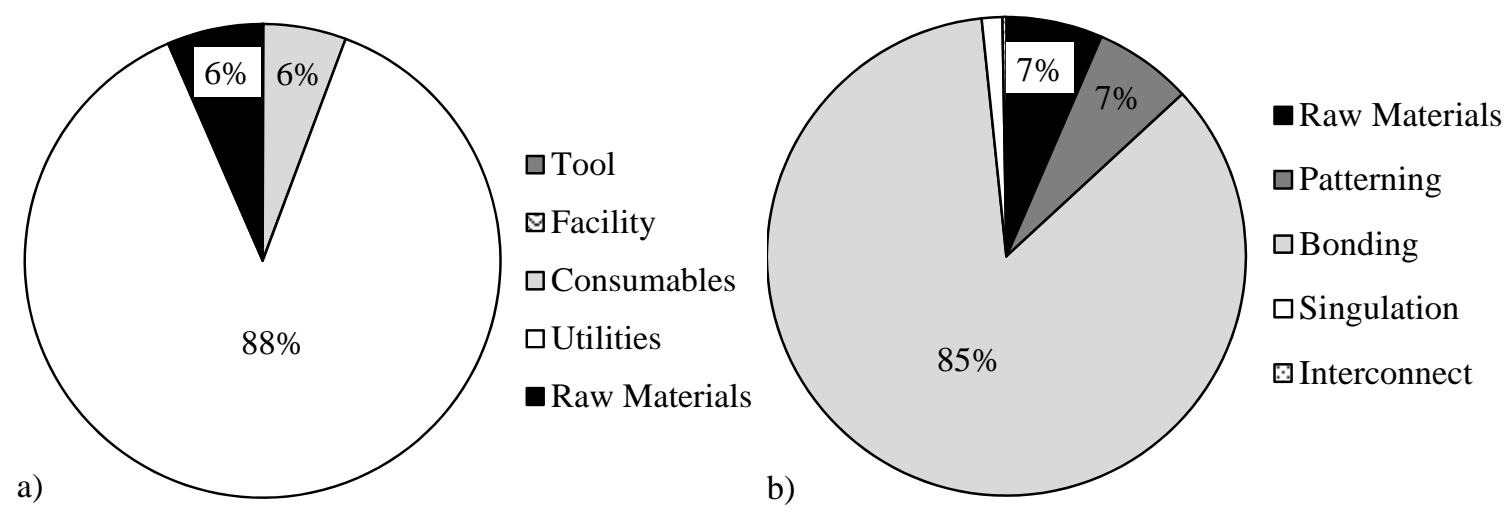

Figure 7. Environmental impact score breakdown by a) Category and by b) Process type and raw material at a production rate of 500,000 HRUs per year

By considering the results of cost and environmental impact modeling, it can be seen that diffusion bonding is both the top cost driver and the top environmental impact driver. Among the cost and environmental impact categories, utilities contribute to half of the cost and most of environmental impacts. This indicates that shorter bonding time and less energy consumption needs to be realized to achieve more economically viable and environmentally responsible 
bonding processes. Thus, new materials and bonding methods must also emerge to address process limitations. At an annual production volume of 1,000 units, the interconnect process is the next most significant cost driver, and accounts for $24.4 \%$ of the total cost, however, its environmental impact is low. PCM ranks as the third cost driver and second environmental impact driver at this production volume, with $17.05 \%$ of cost and $8.64 \%$ of environmental score. Among the cost and environmental impact categories, consumables are the main contributor for both cost and environmental impacts, which indicates a more economically viable and environmentally responsible patterning process should employ a process that reduces consumables use or implements other types of consumable materials and components. The next most significant cost and environmental impact driver is raw material, which accounts for $14.8 \%$ of the cost and $6.3 \%$ of environmental impacts. This indicates less costly and environmentally impactful raw materials and materials processing technologies are needed. As production rate increases, the unit costs of PCM and EDM decreases, while environmental impact is not as sensitive to production volume. Thus, for production volumes of 2,000 or larger, raw materials become the second highest cost driver. The overall results show that to achieve lower cost and less environmentally impactful HRU production, changes are needed in shim patterning and bonding process technology and materials.

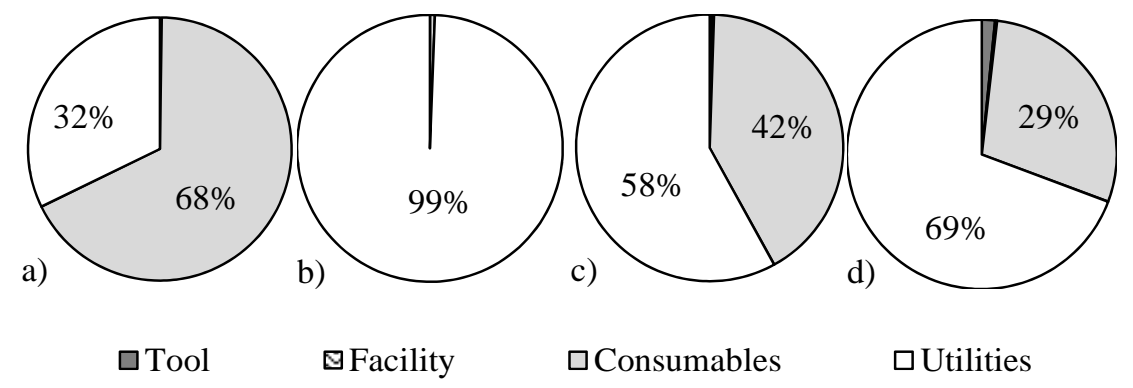

Figure 8. Key sources of environmental impacts for a) Patterning, b) Bonding, c) Singulation, and d) Interconnect processes

\section{Conclusions}

A combined process-based economic and environmental assessment model was developed and used to demonstrate the ability to identify process development strategies for simultaneously reducing the cost and environmental impact of a microchannel heat recovery unit (HRU). As expected, the analysis showed that for a given process plan, the cost and environmental impact per unit device decrease with an increase in production volume. At low production volumes, tools, labor, raw materials and consumables are the top cost drivers, while at high production volumes, raw materials, consumables, and tools are found to be the main cost contributors. In terms of environmental impacts, utilities are the main driver for each production volume. More specifically, it was found that the diffusion bonding process used for microchannel device manufacturing is most important for both cost and environmental impact. To improve the manufacturing process from both cost and environmental perspectives, a bonding process with shorter cycle time and less water and electricity consumption is needed.

The model proposed in this paper establishes a framework for assessment of economic and environmental impacts for manufacturing planning, however, limitations of this work remain. First, for economic assessment, factors such as interest, taxes, profits, and management costs are not considered, which could be included in future model refinement. Also, the cost of raw materials and consumables are independent of production volume, which is a simplifying assumption. Although labor and maintenance are considered as cost categories, they are not completely addressed in the environmental impact assessment model. Tool replacement parts are considered as consumables due to their relatively shorter lifetime compared to the tool itself. The environmental impact of maintenance is not considered. For the case study, environmental impact modeling assumes that the tools are made only of iron and steel. For tools with unknown weight, weights were calculated based on footprint and a density assumption of $150 \mathrm{~kg} / \mathrm{m}^{2}$. Finally, use of the model to assess the difference between manufacturing processes is needed for validating the capacity of the model to choose between manufacturing alternatives. The modeling framework presents a novel approach to simultaneously consider manufacturing costs and environmental impacts in the concurrent design of advanced technology products and dedicated manufacturing facilities to achieve commercialization. 


\section{Acknowledgements}

The authors wish to gratefully acknowledge the support of this research by the U.S. Army (W15P7T08CV201).

\section{References}

Allen, D.M., 2004. Photochemical Machining: from “manufacturing's best kept secret” to a $\$ 6$ billion per annum, rapid manufacturing process. CIRP Ann. - Manuf. Technol. 53, 559-572. doi:10.1016/S00078506(07)60029-8

Allen, D.M., Jefferies, P., 2006. An Economic, Environment-friendly Oxygen-Hydrochloric Acid Regeneration System for Ferric Chloride Etchants used in Photochemical Machining. CIRP Ann. - Manuf. Technol. 55, 205-208. doi:16/S0007-8506(07)60399-0

Anastaselos, D., Oxizidis, S., Papadopoulos, A.M., 2011. Energy, environmental and economic optimization of thermal insulation solutions by means of an integrated decision support system. Energy Build. 43, 686-694. doi:10.1016/j.enbuild.2010.11.013

Avram, O., Stroud, I., Xirouchakis, P., 2010. A Multi-Criteria Decision Method for Sustainability Assessment of the Use Phase of Machine Tool Systems. Int. J. Adv. Manuf. Technol. 53, 811-828. doi:10.1007/s00170-0102873-2

Bradley, D.A., Roman, F., Bras, B., Guldberg, T.A., 2006. A design decision support model for estimating environmental impacts and costs in manufacturing, in: ASME 2006 International Design Engineering Technical Conferences and Computers and Information in Engineering Conference. Philadelphia, Pennsylvania, USA, pp. 727-738. doi:10.1115/DETC2006-99045

Brown, M.O., Haapala, K.R., Eluri, R.T., Paul, B.K., Leith, S.D., King, D.A., 2011. Environmental Impacts of Microchannel Air Preheater Manufacturing under Different Scenarios, in: Proceedings of the IIE Annual Conference and Expo 2011 (IERC 2011). Presented at the IIE Annual Conference and Expo 2011 (IERC 2011), Reno, NV.

Choi, A.C.K., Kaebernick, H., Lai, W.H., 1997. Manufacturing Processes Modelling for Environmental Impact Assessment. J. Mater. Process. Technol. 70, 231-238. doi:16/S0924-0136(97)00067-8

Eastlick, D.D., Haapala, K.R., 2012. Increasing the utility of sustainability assessment in product design, in: ASME 2012 International Design Engineering Technical Conferences \& Computers and Information in Engineering Conference. Presented at the ASME 2012 International Design Engineering Technical Conferences \& Computers and Information in Engineering Conference, American Society of Mechanical Engineers, pp. 713-722.

Environmental Service, 2013. FY 2014 Sewer System Rate Study [WWW Document]. http://www.portlandoregon.gov/bes/, last accessed April 11, 2015.

Fratila, D., 2010. Macro-Level Environmental Comparison of Near-Dry Machining and Flood Machining. J. Clean. Prod. 18, 1031-1039. doi:10.1016/j.jclepro.2010.01.017

Goedkoop, M., Heijungs, R., Huijbregts, M., Schryver, A.D., Struijs, J., Zelm, R., 2009. ReCiPe 2008. PRé Consultants.

Gutowski, T., Murphy, C., Allen, D., Bauer, D., Bras, B., Piwonka, T., Sheng, P., Sutherland, J., Thurston, D., Wolff, E., 2005. Environmentally benign manufacturing: observations from Japan, Europe and the United States. J. Clean. Prod. 13, 1-17. doi:16/j.jclepro.2003.10.004

Haapala, K.R., Tiwari, S.K., Paul, B.K., 2009. An Environmental Analysis of Nanoparticle-Assisted Diffusion Brazing, in: 2009 ASME International Manufacturing Science and Engineering Conference. Presented at the MSEC2009, West Lafayette, Indiana, USA.

Haapala, K.R., Zhao, F., Camelio, J., Sutherland, J.W., Skerlos, S.J., Dornfeld, D.A., Jawahir, I.S., Clarens, A.F., Rickli, J.L., 2013. A Review of Engineering Research in Sustainable Manufacturing. J. Manuf. Sci. Eng. 135, 041013-1 - 041013-16. doi:10.1115/1.4024040

ISO, 2006. ISO 14040:2006, Environmental Management - Life Cycle Assessment - Principles and Framework. International Organization for Standardization.

Lajevardi, B., Leith, S., King, D., Paul, B., 2011. Arrayed Microchannel Manufacturing Costs for an Auxiliary Power Unit Heat Exchanger, in: Proceedings of the 2011 Industrial Engineering Research Conference. Reno, NV.

Leith, S.D., King, D.A., Paul, B., 2010. Toward Low-Cost Fabrication of Microchannel Process Technologies - Cost Modeling for Manufacturing Development, in: Proceedings of the 2010 AIChE Annual Meeting. Salt Lake City, Utah. 
Liow, J.L., 2009. Mechanical Micromachining: a sustainable micro-device manufacturing approach? J. Clean. Prod. $17,662-667$.

Mehendale, S.S., Jacobi, A.M., Shah, R.K., 2000. Fluid Flow and Heat Transfer at Micro- and Meso-Scales With Application to Heat Exchanger Design. Appl. Mech. Rev. 53, 175-193. doi:10.1115/1.3097347

Modica, F., Marrocco, V., Copani, G., Fassi, I., 2011. Sustainable Micro-Manufacturing of Micro-Components via Micro Electrical Discharge Machining. Sustainability 3, 2456-2469.

Munoz, A.A., Sheng, P., 1995. An Analytical Approach for Determining the Environmental Impact of Machining Processes. J. Mater. Process. Technol. 53, 736-758. doi:10.1016/0924-0136(94)01764-R

Paul, B.K., Peterson, R.B., 1999. Microlamination for Microtechnology-based Energy, Chemical, and Biological Systems, in: ASME IMECE. Nashville, TN, pp. 45-52.

Portland General Electric, 2013. Medium and Large Business Energy Price - Market Value of Energy [WWW Document]. http://www.portlandgeneral.com/business/medium_large/energy_pricing/prices/default.aspx. last accessed November 17, 2013.

Portland Water Bureau, 2013. Water volume charges [WWW Document]. http://www.portlandoregon.gov/water/article/27449, last accessed November 17, 2013.

Roy, R., Allen, D., Zamora, O., 2004. Cost of photochemical machining. J. Mater. Process. Technol. 149, 460-465. doi:10.1016/j.jmatprotec.2004.01.059

Salary.com, 2013. Manufacturing Technician II [WWW Document]. Salary.com. URL http://swz.salary.com/SalaryWizard/Manufacturing-Technician-II-Salary-Details-Portland-OR.aspx, last accessed November 17, 2013.

Tan, X.C., Liu, F., Cao, H.J., Zhang, H., 2002. A decision-making framework model of cutting fluid selection for green manufacturing and a case study. J. Mater. Process. Technol. 129, 467-470. doi:10.1016/S09240136(02)00614-3

Weinert, K., Inasaki, I., Sutherland, J.W., Wakabayashi, T., 2004. Dry Machining and Minimum Quantity Lubrication. CIRP Ann. - Manuf. Technol. 53, 511-537. doi:16/S0007-8506(07)60027-4

Winter, M., Li, W., Kara, S., Herrmann, C., 2014. Determining optimal process parameters to increase the ecoefficiency of grinding processes. J. Clean. Prod. 66, 644-654. doi:10.1016/j.jclepro.2013.10.031

Zhang, H., Haapala, K.R., 2014. Integrating sustainable manufacturing assessment into decision making for a production work cell. J. Clean. Prod. doi:10.1016/j.jclepro.2014.01.038

Zhao, F., Murray, V.R., Ramani, K., Sutherland, J.W., 2012. Toward the development of process plans with reduced environmental impacts. Front. Mech. Eng. doi:10.1007/s11465-012-0334-3 
Appendix A. Graphical user interface: Basic user form

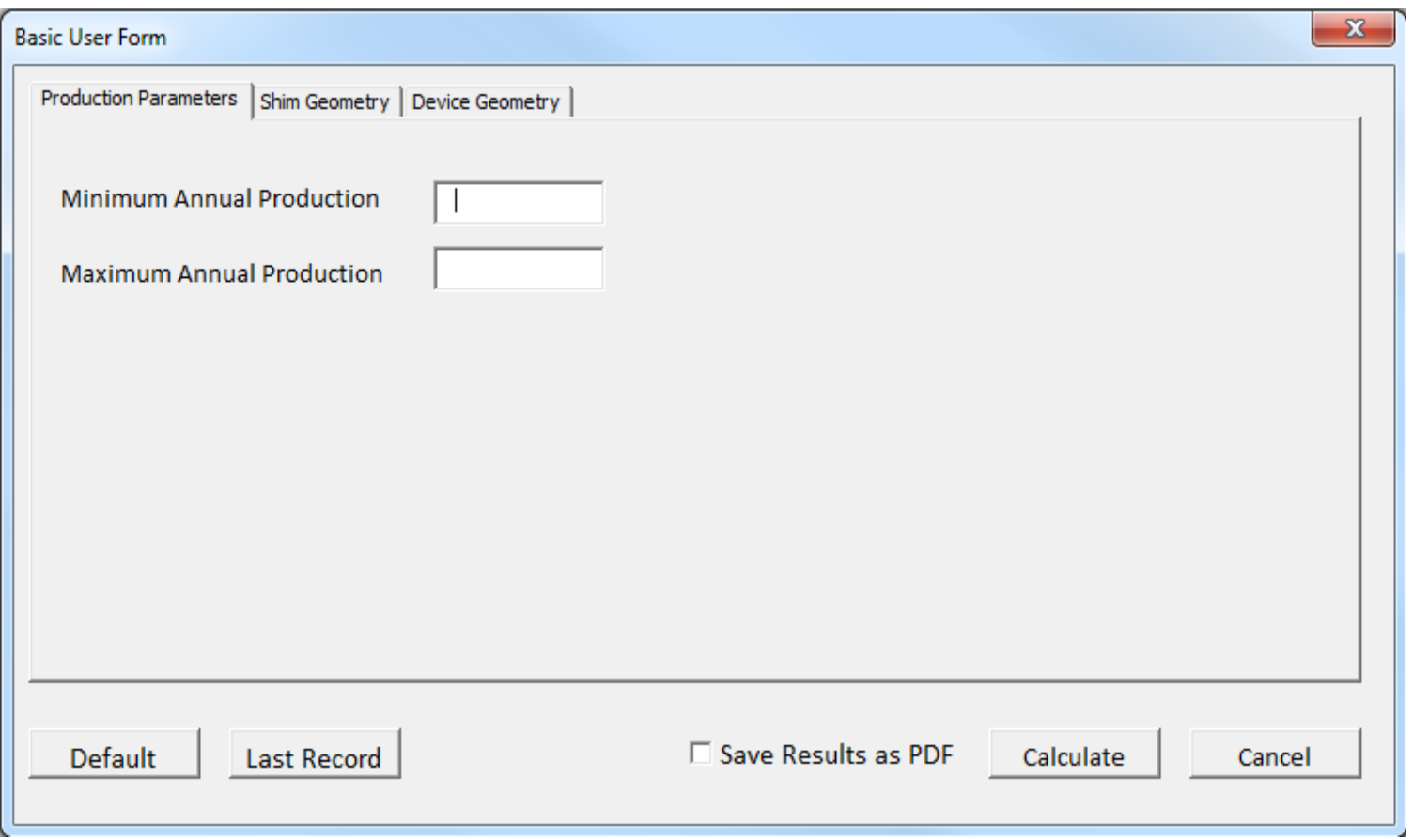

Figure A.1. Tab to enter production parameters

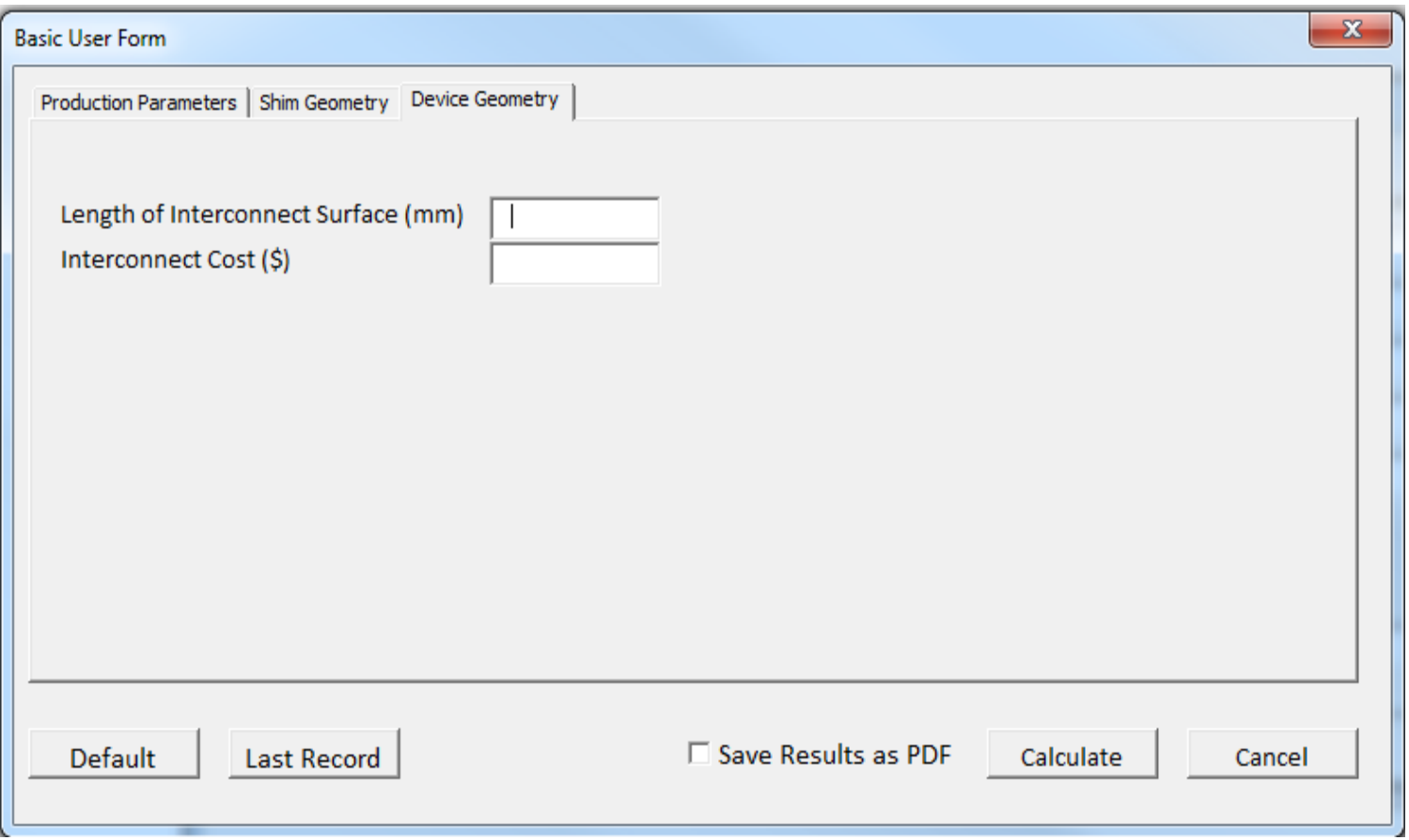

Figure A.2. Tab to enter device geometry information 


\section{Figure captions}

Figure 1. Contributors to product manufacturing costs

Figure 2. Graphical user interface for shim geometry data entry

Figure 3. Heat recovery unit (HRU) design

Figure 4. General manufacturing process flow for the heat recovery unit (HRU)

Figure 5. Cost breakdown by a) Cost category and b) Process type and raw material

Figure 6. Key cost categories for a production volume of 20,000 devices/year: a) Patterning, b) Bonding, c) Singulation, and d) Interconnect processes

Figure 7. Environmental impact score breakdown by a) Category and by b) Process type and raw material at a production rate of 500,000 HRUs per year

Figure 8. Key sources of environmental impacts for a) Patterning, b) Bonding, c) Singulation, and d) Interconnect processes

Figure A.1. Tab to enter production parameters

Figure A.2. Tab to enter device geometry information 